Rev. Elev. Méd. vét. Pays trop., 1975, 28 (1) : 79-82

\title{
Note sur la phénologie des graminées de savane
}

\author{
par P. GRANIER $\left({ }^{*}\right)$, Y. CABANIS
}

\begin{abstract}
RESUME
Les observations sur la période de reproduction de seize espèces de graminées des savanes des Hauts-Plateaux malgaches ont montré un échelonnement des floraisons pendant toute la saison des pluies, une étroite relation entre la précocité de la floraison et la durée du cycle de reproduction, une plus grande précocité de certains types morphobiologiques, des espèces pérennes à feuilles basilaires en particulier.

Les caractéristiques des cycles de reproduction peuvent être considérées comme des critères d'adaptation des espèces à leur milieu et les auteurs pensent pouvoir les utiliser pour approfondir la connaissance du déterminisme des associations végétales des savanes de Madagascar.
\end{abstract}

La savane est la formation herbacée la mieux représentée à Madagascar. Elle couvre 35 millions d'hectares environ et s'étend sur plusieurs régions bioclimatiques. Des variations, d'ordre floristique, physionomique, structural, phytosociologique, interviennent nécessairement si l'on progresse le long d'une catena topographique qui va des Hauts Plateaux jusqu'à la mer. Pourtant, malgré des différences d'aspect dues à des raisons écologiques diverses, la savane reste fondamentale et comparable en tous points de l'Ile. Elle est caractérisée par :

- Un recouvrement global dense si la végétation n'est pas dégradée par l'homme;

- Une pauvreté numérique en espèces et en contrepartie une constance de ces espèces (Hyparrhenia rufa, Aristida rufescens, Imperata cylindrica, Heteropogon contortus);

- Une origine le plus souvent étrangère de graminées à grande extension (africaines);

- Une abondance de vivaces du type biologique : Hémicryptophyte pérenne à floraison annuelle, une rareté des thérophytes et la présence de 2 géophytes;

Région de Recherches Vétérinaires et Zootechniques de Madagascar.

(*) Laboratoire de l'Elevage, B.P. 485, Niamey, République du Niger.
- L'absence de ligneux savanicoles sur les formations herbacées des plateaux où le sol est ferrallitique;

- L'apparition d'espèces dominantes ayant une signification écologique précise;

- Un dynamisme actif introduisant une évolution rapide des faciès et un retour au peuplement fermé.

Dans un milieu où le facteur limitant est l'eau, s'établit entre les végétaux appartenant à la même strate aérienne et aux mêmes horizons pédologiques, une concurrence inévitable qui oblige chaque espèce à tirer le meilleur parti des ressources du sol.

Peu d'indications nous sont acquises sur les adaptations constatées. L'époque de floraison nous a paru le critère d'observation intéressant pour caractériser les cycles du peuplement graminéen.

Les observations faites dans la région de Tananarive sont applicables à la région des plateaux correspondante (altitude supérieure à $1000 \mathrm{~m}$ ). Les caractéristiques du milieu (dont nous donnons la description précise en annexe) sont l'existence d'une très longue saison sèche (7 mois environ) d'une forte pente et d'un drainage maximal. Les sols ferrallitiques saturés 
en période humide, perdent leur humidité rapidement en surface par suite de l'absence ou de la réduction de l'horizon humifère.

La végétation de ces collines dont les pentes sont très accidentées est une savane basse mais fermée si l'influence de l'homme n'est pas trop aggravante. Aristida rufescens géophyte cespiteuse cauliphylle y domine. Les associées sont de 2 types, des Hémicryptophytes cespiteuses basiphylles (Elionurus, Craspedorachis...) ou cespiteuses cauliphylles (Hyparrhenia, Cymbopogon). La présence de ces groupes d'espèces apporte une indication précieuse sur le stade d'évolution du milieu. En effet, si l'apparition du premier type biomorphologique correspond à un appauvrissement du sol, la présence du deuxième caractérise un support mieux structuré et plus riche en humus (zones planes ou d'accumulation).

Les observations consignées dans le tableau permettent de suivre le comportement de chaque espèce et de délimiter sa période de reproduction (de la floraison à la dissémination). La comparaison de ces différents cycles montre:

1. Un étalement des floraisons pendant toute la saison des pluies: Certaines se produi- sant d'une manière précoce (1 à 2 mois après les premières précipitations), les autres au-delà de la limite des pluies. On peut ainsi distinguer des espèces à cycle court (Elionurus, Alloteropsis, Andropogon...) et des espèces à cycle long (Hyparrhenia, Aristida...);

2. Une durée du cycle de reproduction en relation avec la précocité de floraison: Les premières espèces à fleurir accomplissent toutes les étapes de la formation des graines en moins d'un mois. $\mathrm{Au}$ contraire, les espèces tardives ont un cycle plus lent et plus étalé ( 3 mois et plus pour Aristida);

3. Une succession échelonnée des mises à fleur des différentes espèces : Il est remarquable de constater la répartition des floraisons tout le long de la saison des pluies ou à certaines périodes de cette saison. C'est ainsi que pendant le mois de janvier (du 20-12 au 20-1), 4 espèces de la même station fleurissent successivement avec un décalage de 8 jours entre elles;

4. Une précocité particulière de certains types morphologiques: Les premières floraisons se trouvent sur les espèces pérennes à feuilles basilaires. Au contraire, les plantes à feuilles caulinaires et à appareil végétatif bien déve-

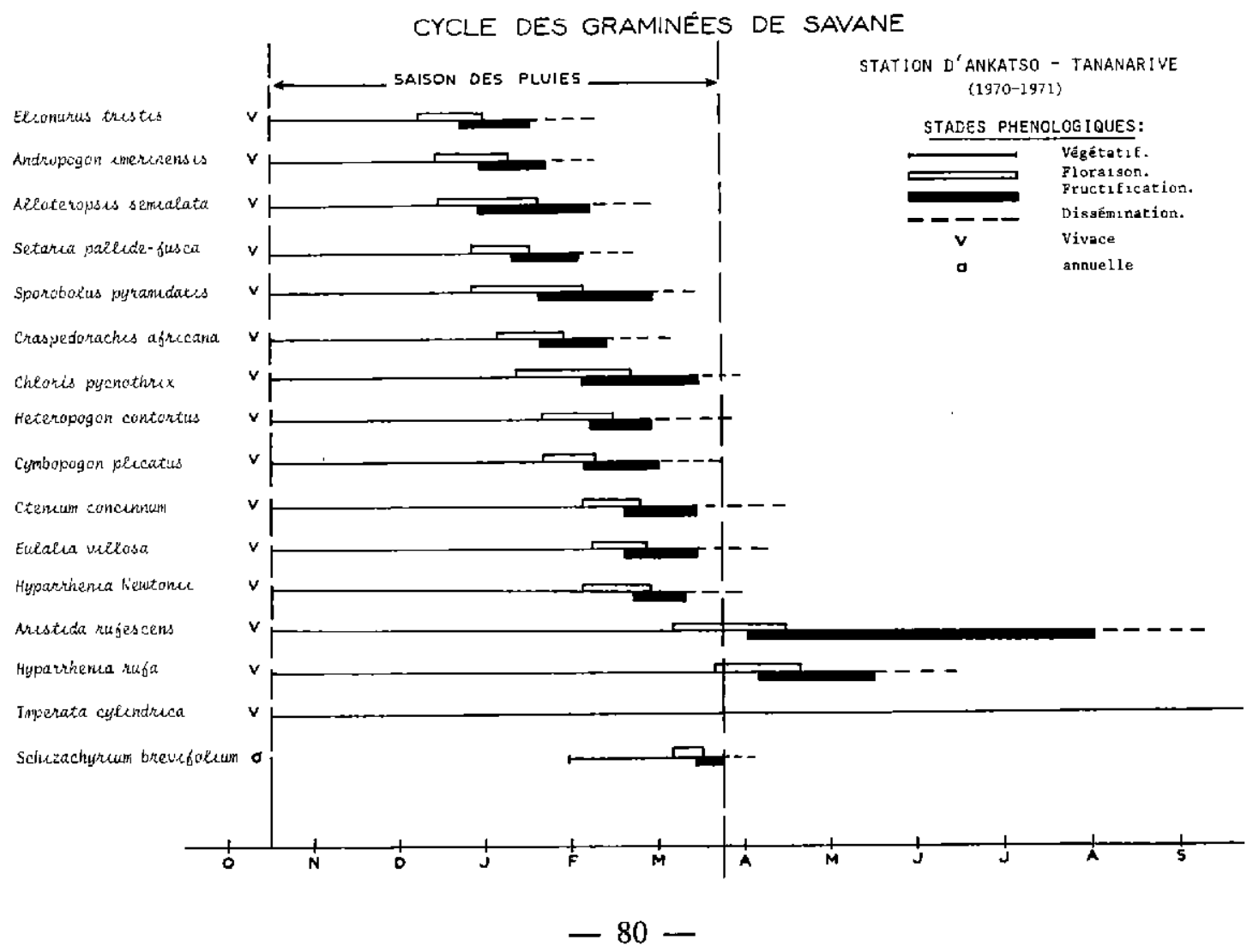


loppé sont plus tardives et poursuivent leur cycle jusqu'à la fin de la saison des pluies.

Une annuelle à type morphologique uniculmaire, donc à système végétatif réduit, a un cycle très court et une floraison rapide.

\section{REMARQUES SUR LE GRAPHIQUE DES CYCLES}

1. La saison des pluies, commencée dans ia deuxième quinzaine d'octobre a duré 5 mois. Elle doit être considérée comme une année déficitaire (1 $181 \mathrm{~mm}$ soit $179 \mathrm{~mm}$ de moins que la moyenne) et à répartition irrégulière. Nous avons cependant pris comme point de départ les premières précipitations.

2. La période de floraison commence aux environs du 20-12, soit 2 mois après le début des pluies et se termine vers le 20 mars pour les dernières espèces, soit 5 mois après le début des pluies. Les floraisons des diverses espèces sont étalées sur 3 mois.

3. Le temps compris entre le début des pluies et la floraison correspond au cycle végétatif. Nous avons classé les espèces selon la durée de ce cycle :

- 1 à 2 mois : Elionurus, Andropogon, Alloteropsis, Schizachyrium.

- 2 à 3 mois : Craspedorachis, Setaria, Sporobolus.

- 3 à 4 mois : Eulalia, Ctenium, Cymbopogon, Heteropogon.

\section{- 4 à 5 mois: Hyparrhenia, Aristida.}

Les précédentes observations permettent d'envisager le problème de l'adaptation des graminées de savane à leur environnement et d'entrevoir à quel niveau se situe la concurrence interspécifique. Il nous paraît intéressant de souligner les points suivants:

D'abord, la présence de plusieurs types biomorphologiques dans les peuplements savanicoles est une réponse aux variations du milieu. On peut remarquer qu'à chaque type correspond en général un stade d'évolution du support.

- Hemicryptophyte cespiteux basiphylle sur sol à horizon humifère réduit.

- Hemicryptophyte cespiteux cauliphylle sur sol plus profond et plus évolué.
Ensuite, l'existence d'espèces différentes d'autant plus nombreuses que le milieu devient plus rigoureux est une preuve d'une sélection naturelle des génotypes les mieux adaptés. Celle-ci, par le jeu de la compétition interspécifique, a forcé chaque espèce à utiliser le temps et la place laissés libres par les concurrentes. La succession des cycles de reproduction et les durées de chaque cycle végétatif sont en rapport avec la nature du sol, sa richesse et les disponibilités en eau. Les plantes à cycle court auraient des besoins moindres et un développement réduit par suite d'une longue adaptation à des circonstances rigoureuses. En d'autres termes, moins finalistes, on peut dire que, seules parmi les espèces, celles qui ont pu se reproduire dans ces conditions précises, ont permis la création de populations bien en place dans le milieu et nécessairement compétitives.

La présence de types biomorphologiques particuliers et d'espèces sélectionnées permet de se faire une idée des conditions d'une station ou d'un milieu. L'existence de plantes dominantes constitue une précieuse indication sur le stade d'évolution d'un peuplement. La connaissance plus approfondie des besoins de chaque plante est nécessaire pour mieux saisir le sens des variations. Nous avons mis l'accent sur la valeur du support et sur l'approvisionnement en eau qui représente le facteur limitant le plus impératif. Si la température et ses variations ne paraissent pas avoir une influence prépondérante, par contre il n'est pas exclu que le photopériodisme puisse intervenir en favorisant la floraison de certaines espèces pendant les jours les plus longs (décembre).

Enfin, en ce qui concerne les relevés de végétation et la caractérisation des peuplements savanicoles, l'observation de la végétation en un temps donné entraîne sûrement des interprétations incomplètes ou inexactes à cause de lapparente dominance des espèces en floraison ou en montaison. Il est vraisemblable qu'on ignore l'existence ou la contribution des espèces à cycle différent. N'ayant pas une idée exacte des changements saisonniers de faciès du peuplement savanicole, on risque des erreurs d'interprétation sur la valeur des pâturages ou l'état d'évolution de la formation graminéenne.

Cette note préliminaire est une introduction à des observations plus approfondies contribuant à préciser la nature de la compétition interspécifique et les causes de la sélection. 


\section{ANNEXE}

1. Siruation

\section{Caractéristiques de la Station Tananarive (Ankatso)}

- Altitude $1350 \mathrm{~m}$, exposition Sud-Est

- Pente supérieure à 25 p. 100

- Sols ferrallitiques peu profonds sur granite

2. Données climatiques (saison 70-71)

A. Pluviométrie

\begin{tabular}{|l|cccccccccccc|}
\hline & O & N & D & J & F & M & A & M & J & J & A & S \\
\hline Pluies en $\mathrm{m} / \mathrm{m}$ & 23 & 200 & 147,6 & 330,4 & 288,9 & 144,2 & 4,9 & 8,5 & 0,4 & 3,8 & 3,9 & 26,4 \\
\hline Nbre de jours & 2 & 18 & 16 & 23 & 21 & 8 & 2 & 6 & 2 & 4 & 8 & 4 \\
\hline
\end{tabular}

Hauteur des précipitations : $1181,8 \mathrm{~mm}$

B. Températures

\begin{tabular}{|l|cccccccccccc|}
\hline & O & N & D & J & F & M & A & M & J & J & A & S \\
\hline Moyenne max. & 27,4 & 26,6 & 26,1 & 25,3 & 26,2 & 26,2 & 26,1 & 23,9 & 20,4 & 20,4 & 21,1 & 23,9 \\
\hline Moyenne min. & 12,3 & 14,6 & 14,3 & 15,6 & 15,5 & 14,7 & 14,3 & 11,9 & 8,8 & 9,3 & 8 & 9,7 \\
\hline
\end{tabular}

C. Evaporimétrie

\begin{tabular}{|c|cccccccccccc|}
\hline & O & N & D & J & F & M & A & M & J & J & A & S \\
\hline $\begin{array}{c}\text { Moyenne } \\
\text { mensuelle }\end{array}$ & 124,3 & 75,2 & 77,2 & 63,6 & 62,7 & 66,5 & 73 & 76,8 & 65,4 & 65,5 & 76,9 & 98,5 \\
\hline
\end{tabular}

\section{Exploitation}

Mise à feu périodique et pâturage irrégulier.

\section{SUMMARY}

\section{Note on the phenology of savanna grasses}

Observations on the reproductive phase of sixteen savanna graminaceous species on the Malagasy Plateaux showed staggered flowering throughout the rainy season, a close relationship between flowering earliness and the duration of the reproductive phase and some earlier morphobiological types, more particular in the perennial species with basilar leaves.

The characteristics of the reproductive cycle can be considered as criteria of species adaptation to their environment and the authors think that they can be used to have a deeper knowledge of the determinism of savanna plant association in Madagascar.

\section{RESUMEN}

\section{Nota sobre la fenologia de las gramíneas de sabana}

Las observaciones sobre el periodo de reproducción de diez y seis especies de gramineas de las sabanas de las Altiplanicies malgachas mostraron un escalonamiento de las floraciones durante la estación de las lluvias, una relación estrecha entre la precocidad de la floración y la duración del ciclo de reproducción, una precocidad más grande de ciertos tipos morfobiologicos, particularmente de las especies perennes con hojas basilares.

Se pueden considerar las características de los ciclos de reproducción como criterios de adaptación de las especies a su medio y los autores piensan poder utilizarlas para conocer mejor el determinismo de las asociaciones vegetales de las sabanas de Madagascar. 